SHORT REPORT

\title{
Phenotypic variability in siblings with type III spinal muscular atrophy
}

\author{
M M K Muqit, J Moss, C Sewry, R J M Lane
}

J Neurol Neurosurg Psychiatry 2004;75:1762-1764. doi: 10.1136/jnnp.2003.018614

Autosomal recessive spinal muscular atrophy (SMA) shows substantial phenotypic variability, presenting at a variety of ages from infancy to adult life. Diagnostic difficulties may arise because SMA sometimes produces a dystrophic or myopathic phenotype rather than classical neurogenic abnormalities. Two brothers are described who illustrate this principle and highlight the increasing importance of molecular genetics in investigating patients with neuromuscular diseases. The findings are discussed in the light of recent observations in a mouse model of SMA.

$\mathrm{S}$ pinal muscular atrophy (SMA) is characterised by progressive degeneration of anterior horn cells. Four subtypes of the common autosomal recessive form are recognised, based on age at onset of symptoms and clinical course. ${ }^{1}$ Type I SMA (Werdnig-Hoffmann disease) is the most severe, with weakness developing within the first six months of life and death within two years. In type II SMA, weakness usually begins between three and 15 months of age. These children never manage to stand or walk unassisted but they survive into adolescence. In type III SMA (Kugelberg-Welander disease), onset is usually after the second year of life and progression is variable. The disease is characterised by proximal weakness, predominantly of the legs. Patients typically have normal milestones in the first year of life and manage to stand and walk unaided but have problems with running and jumping. They usually maintain ambulation over many years and the prognosis is generally good. Type IV (adult onset) SMA usually presents with a limb girdle phenotype in the third decade. ${ }^{2}$

Autosomal recessive SMA is caused by mutations in the telomeric copy of the survival motor neurone gene (SMN-1) at 5q11.1-13.3. ${ }^{3}$ Deletions of exon 7 and 8, or 7 alone, account for over $95 \%$ of SMA cases. ${ }^{3}$ Most of the remaining cases have intragenic point mutations in the SMN-1 gene. The molecular mechanisms by which SMN-1 mutations cause SMA remain unknown. Other genes at the SMA locus have been reported as candidate modifiers for the SMA phenotype, of which SMN-2 is thought to be the most important. ${ }^{4}$

Intrafamilial phenotypic variation in SMA is rare. Previous reports have described differences in severity within the same family. ${ }^{5}$ In contrast we report two brothers with identical homozygous deletions of exons 7 and 8 of SMN-1 but with strikingly different pathological phenotypes. We highlight the importance of molecular genetics as part of the routine investigation of neuromuscular disorders and discuss a possible molecular basis for the discordant phenotypes of SMA in the light of recent findings in mouse models. ${ }^{67}$

\section{CASE HISTORIES}

\section{Patient 1}

A 16 year old boy first became aware of proximal leg weakness about one year before his referral. He had achieved normal motor milestones and managed sports at school. He had noticed some thinning of his thighs and a fine tremor of his hands and head. He had not suffered muscle pains or cramps. His parents were first cousins and he had a five year old brother and four sisters. The brother (patient 2) was also eventually found to have neuromuscular problems but the sisters were asymptomatic.

On examination he had mild neck flexion weakness and normal extension. There was generalised muscle wasting with severe symmetrical weakness, affecting proximal more than distal musculature. There was no fasciculation. His calves were not hypertrophied. Reflexes in the upper limbs were all reduced and the knee jerks were absent, but the ankle jerks were brisk. He had a fine tremulousness of his limbs and head, and showed a typical Gowers manoeuvre. His gait was normal and he could hop on either foot.

Serum creatine kinase (CK) was increased to $1181 \mathrm{U} / \mathrm{l}$ (0-220). Nerve conduction studies were normal. Quantitative electromyography of the right vastus lateralis showed no fibrillations or fasciculations, but the motor units were of short duration and reduced size, suggesting a chronic myopathic process. Muscle biopsy from the left quadriceps (fig lA) showed myopathic changes, with variation in fibre size, a significant increase in frequency of internal nuclei, and occasional necrotic fibres. None of the features commonly associated with neurogenic disorders was observed. HLA staining was negative except on necrotic fibres and some occasional small (regenerating) fibres. Dystrophin staining was normal and DNA analysis showed no deletions in the dystrophin gene. However, there was homozygous deletion of exons 7 and 8 of the telomeric copy of the SMN gene, confirming a diagnosis of SMA.

The patient was encouraged to undertake a weight training programme combined with a high protein diet. At one year follow up his muscle strength and function had remained stable.

\section{Patient 2}

This five year old boy was examined at the time of his elder brother's evaluation. He had no specific symptoms and his father did not feel that he was lagging behind his peers in sports at school. However, he ran in a rather flat footed fashion, had difficulty hopping, and showed a partial Gowers manoeuvre. He had some neck weakness and mild proximal weakness in both upper and lower limbs, with preserved distal strength. His calves were not hypertrophied. He had diminished knee jerks and intact ankle jerks. All his blood results were normal, including CK.

His muscle biopsy (fig lB) showed frequent atrophic angular fibres that stained intensely with oxidative enzymes, but there was no evidence of fibrosis, inflammation, or

Abbreviations: SMA, spinal muscular atrophy; SMN, survival motor neurone 

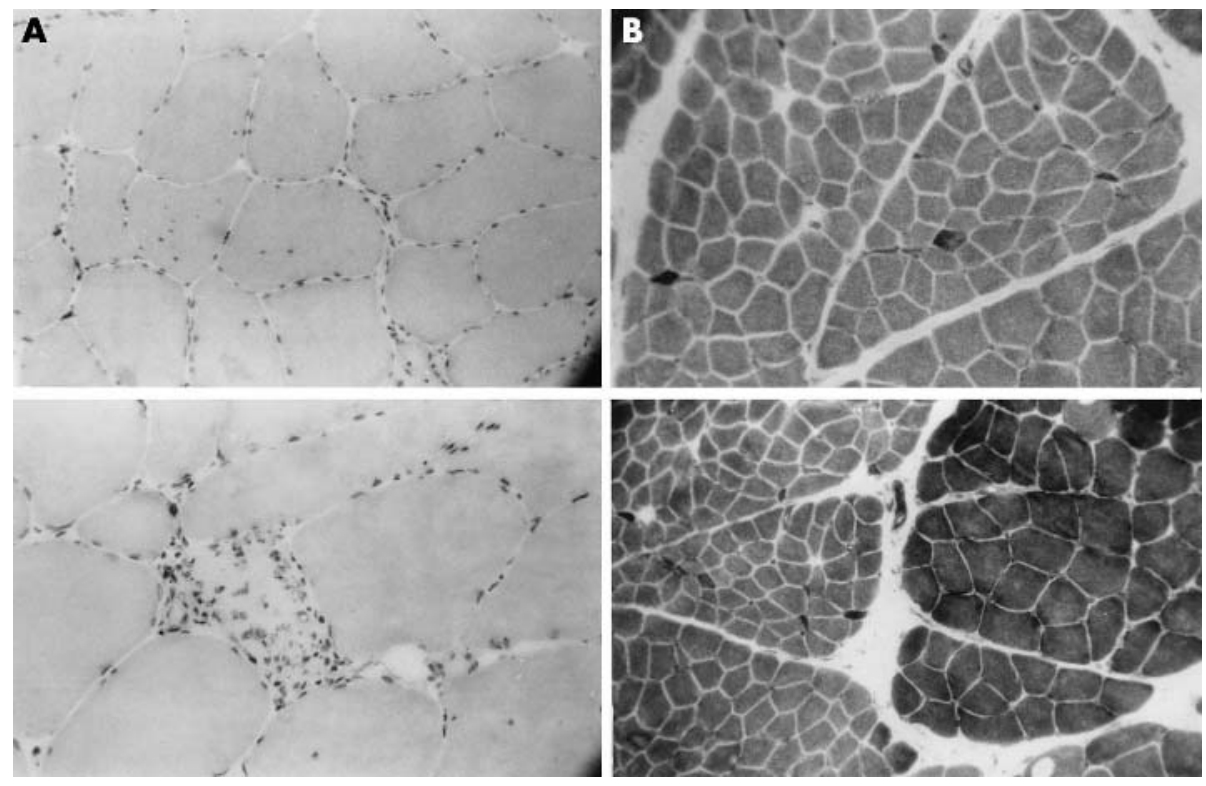

Figure 1 (A) Patient 1. Top panel (haematoxylin and eosin stain): there is increased variation in fibre sizes, with hypertrophy of some fibres (histochemically type 2) and an increase in internal nuclei. Lower panel (acid phosphatase stain): there are frequent, scattered fibres undergoing necrosis, with granular cytoplasm and marked acid phosphatase staining indicating macrophage infiltration. (B) Patient 2. Top panel (NADH-TR stain): scattered, intensely staining, angulated fibres are seen. In contrast to patient 1, no necrotic fibres were seen and there was no increase in internal nuclei. Lower panel (NADH-TR stain): marked fibre type grouping is evident, but there is no grouped atrophy.

necrosis. Histochemistry revealed fibre type grouping, indicating denervation with reinnervation, but no grouped fibre atrophy. On genetic analysis there were no deletions in the dystrophin gene, but, like his older brother, he had homozygous deletions of exons 7 and 8 of the telomeric copy of the SMN gene.

At one year follow up he was able to run relatively well but continued to have difficulty rising from the supine position.

\section{DISCUSSION}

Homozygous deletions or mutations of exon 7 of the SMN1 gene have been found in more than $95 \%$ of SMA cases. ${ }^{3}$ Both our patients achieved normal milestones in the first year of life but developed symptoms or signs after the age of two years, consistent with type III SMA. ${ }^{1}$ However, the diagnosis of type III SMA was not immediately apparent in the proband (patient 1) as his laboratory abnormalities were more in keeping with a muscular dystrophy or myopathy. By contrast, his younger brother (patient 2) had a normal serum CK and histopathological abnormalities consistent with classical SMA.

About a quarter of type III SMA patients are reported to have a dystrophic phenotype with high serum CK levels and "myopathic" histopathology. ${ }^{4}$ While there have been previous reports of affected siblings having different degrees of clinical severity, ranging from type I to type III, ${ }^{5}$ there have been no reports of siblings with the same SMN-1 mutation having discordant clinical and pathological phenotypes of type III SMA.

The biological factors determining a dystrophic rather than the classical neurogenic phenotype are unknown. In patient 1 , there was no suggestion of an immune reaction because there was no upregulation of MHC expression on the nonnecrotic fibres. Phenotypic variation in SMA might reflect genetic factors. Three other genes within the SMA locusnamely, neuronal apoptosis inhibitory protein (NAIP), H4F5, and SMN-2-have been reported to modify the SMA phenotype. ${ }^{4}$ However, SMN-2 is thought to be most important as the amount of SMN protein expressed in tissues appears to be inversely correlated with clinical severity. ${ }^{8}$

Recently, a conditional transgenic mouse model of SMA has been reported. The investigators showed that if exon 7 of the mouse orthologue of SMN-1, smn, is deleted in neurones, affected mice die prematurely and have severe muscle denervation. ${ }^{6}$ However, if exon 7 of $s m n$ is deleted in skeletal muscle, the mice die prematurely and have muscle necrosis and a dystrophic phenotype. ${ }^{7}$ Thus the varying phenotypes in our siblings might reflect differential amounts of SMN protein expression in muscle compared with motor neurones, owing to somatic mosaicism of the SMN-1 gene in these tissues.

Secondary myopathic abnormalities are well recognised in cases of primary denervation and reinnervation. It has been suggested that myopathic changes in muscle biopsies of type III SMA are usually seen only in the later stages of disease ${ }^{9}$ and it is possible that "neurogenic" abnormalities would have been evident in case 1 if the biopsy had been done earlier. However, he was investigated only a few months after the onset of weakness, suggesting that his "myopathic" phenotype is indeed distinct from the "neurogenic" phenotype of his brother. It has also been suggested that there is unlikely to be diagnostic difficulty in distinguishing type III SMA from limb girdle muscular dystrophy ${ }^{10}$ but case 1 shows that this may not hold true. We hope to raise awareness of the potential pitfalls in diagnosing type III SMA and the importance of molecular genetics in the diagnostic repertoire.

\section{Authors' affiliations}

R J M Lane, Division of Clinical Neurosciences and Psychological Medicine, Imperial College at Charing Cross Hospital, London W6, UK J Moss, Division of Pathology, Imperial College at Charing Cross Hospital

C Sewry, Dubowitz Neuromuscular Unit, Imperial College at Hammersmith Hospital, London W12

M M K Muqit, Department of Molecular Pathogenesis, Institute of Neurology, National Hospital for Neurology and Neurosurgery, Queen Square, London WC1

Competing interests: none declared 
Correspondence to: Dr Russell J M Lane, West London Neurosciences Centre, Charing Cross Hospital, Fulham Palace Road, London W6 8RF, UK; r.lane@imperial.ac.uk

Received 15 May 2003

In revised form 18 October 2003

Accepted 8 November 2003

\section{REFERENCES}

1 Munsat TL, Davies KE. International SMA consortium meeting. Meeting report. Neuromuscul Disord 1992;2:423-8.

2 Brahe C, Servidei S, Zappata S, et al. Genetic homogeneity between childhood-onset and adult-onset autosomal recessive spinal muscular atrophy. Lancet 1995;346:741-2.

3 Lefebvre S, Burglen L, Reboullet S, et al. Identification and characterisation of a spinal muscular atrophy-determining gene. Cell 1995;80:155-65.
4 Nicole S, Cifuentes-Diaz C, Frugier T, et al. Spinal muscular atrophy: recent advances and future prospects. Muscle Nerve 2002;26:4-13.

5 Parano E, Pavone L, Falsaperla R, et al. Molecular basis of phenotypic heterogeneity in siblings with spinal muscular atrophy. Ann Neurol 1996:40:247-51.

6 Frugier T, Tiziano FD, Cifuentes-Diaz C, et al. Nuclear targeting defect of SMN lacking the $C$-terminus in a mouse model of spinal muscular atrophy. Hum Mol Genet 2000;9:849-58.

7 Cifuentes-Diaz C, Frugier T, Tiziano FD, et al. Deletion of murine smn exon 7 directed to skeletal muscle leads to severe muscular dystrophy. J Cell Biol 2001;152:1107-14.

8 Lefebvre $S$, Burlet $P$, Liu $Q$, et al. Correlation between severity and SMN protein level in spinal muscular atrophy. Nat Genet 1997;16:265-9.

9 Dubowitz V. Muscle biopsy: a practical approach, 2nd ed. London: Ballière Tindall, 1985

10 Pogue R, Jackson T, Sayli B, et al. Limb-girdle muscular dystrophy or spinal muscular atrophy: a source of diagnostic confusion? J Med Genet 1997;34:958-9. 